Article

\title{
Dynamic Distance Measure on Spaces of Isospectral Mixed Quantum States
}

\author{
Ole Andersson * and Hoshang Heydari \\ Department of Physics, Stockholm University, 10691 Stockholm, Sweden \\ * Author to whom correspondence should be addressed; E-Mail: olehandersson@ gmail.com; \\ Tel.:+46-8-55378614.
}

Received: 18 June 2013; in revised form: 20 August 2013 / Accepted: 2 September 2013 /

Published: 6 September 2013

\begin{abstract}
Distance measures are used to quantify the extent to which information is preserved or altered by quantum processes, and thus are indispensable tools in quantum information and quantum computing. In this paper we propose a new distance measure for mixed quantum states, which we call the dynamic distance measure, and we show that it is a proper distance measure. The dynamic distance measure is defined in terms of a measurable quantity, which makes it suitable for applications. In a final section we compare the dynamic distance measure with the well-known Bures distance measure.
\end{abstract}

Keywords: distance measure; mixed state; quantum dynamics; quantum information

Classification: PACS 03.67.-a; 03.67.Lx; 03.65.Aa; 02.40.Yy; 02.40.Ky

\section{Introduction}

Quantum information has the reputation of being a futuristic field full of far-reaching promises. The field has attracted researchers from many different branches of science and engineering, whose efforts have greatly improved our understanding of the physical nature of information, and hopefully will provide us with new cutting-edge technological innovations in the future. Quantum information theory has been applied to such diverse areas as bio-science, nano-technology, economics, and game theory [1-3].

In recent years, new experimental results have shed light on some murky and hidden parts of quantum information, and have also opened up new opportunities beyond our expectations. Furthermore, new 
theoretical tools, mostly from geometry and topology, have been successfully applied to the field. For example, geometrical considerations led to the important characterization of entanglement and the development of efficient, error-prone systems for quantum computers.

Distance measures are some of the most basic geometrical tools used in quantum information theory. Such measures are, for example, used to compare the input and output of quantum channels and gates, and hence to quantify to what extent information is preserved, or altered, by quantum processes. Examples of well-known distance measures are the trace-distance, fidelity, and Bures distance [4-6]. In this paper we propose a new distance measure that we call the dynamic distance measure. This distance measure is defined for all pairs of isospectral, i.e., unitarily equivalent, mixed states. We show that the dynamic distance is a proper distance measure - a verification that, despite the naturalness of the definition, requires a surprisingly extensive geometric machinery.

Here is the outline of the paper. In Section 2 we define the dynamic distance measure, and state the main result. In Section 3 we introduce a geometric framework, designed and tailored to our needs, and in Section 4 we in detail discuss properties of the dynamic distance measure and prove the main result. Section 5 contains a derivation of the dynamic distance between distinguishable isospectral mixed states. Finally, in Section 6 we compare the dynamic distance measure and the Bures distance.

\section{Isospectral Mixed States and the Dynamic Distance Measure}

Mixed quantum states can be represented by density operators, i.e., self-adjoint, positive, trace-class operators with unit trace. We denote the space of density operators for a quantum system modeled on a Hilbert space $\mathcal{H}$ by $\mathcal{D}(\mathcal{H})$, and its subspace of density operators with finite rank at most $k$ by $\mathcal{D}_{k}(\mathcal{H})$.

A density operator that evolves according to a von Neumann equation remains in a single orbit of the left conjugation action of the unitary group of $\mathcal{H}$ on $\mathcal{D}(\mathcal{H})$. The orbits are in one-to-one correspondence with the possible spectra for density operators on $\mathcal{H}$. By the spectrum of a density operator of rank $k$ we mean its descending sequence $\sigma=\left(p_{1}, p_{2}, \ldots, p_{k}\right)$ of positive eigenvalues, repeated in accordance with their multiplicity. We henceforth assume $\sigma$ to be fixed, and write $\mathcal{D}(\sigma)$ for the corresponding orbit.

Suppose $\rho_{0}$ and $\rho_{1}$ are two density operators in $\mathcal{D}(\sigma)$. Let $H$ be a Hamiltonian operator on $\mathcal{H}$, and assume that a curve $\rho$ satisfies the boundary value von Neumann equation:

$$
i \dot{\rho}=[H, \rho], \quad \rho\left(t_{0}\right)=\rho_{0}, \quad \rho\left(t_{1}\right)=\rho_{1}
$$

We then define the $H$-distance from $\rho_{0}$ and $\rho_{1}$ to be the path integral of the uncertainty of $H$ along $\rho$,

$$
D_{H}\left(\rho_{0}, \rho_{1}\right)=\int_{t_{0}}^{t_{1}} \sqrt{\operatorname{Tr}\left(H^{2} \rho\right)-\operatorname{Tr}(H \rho)^{2}} \mathrm{~d} t
$$

We also define the dynamic distance between $\rho_{0}$ and $\rho_{1}$ to be

$$
D\left(\rho_{0}, \rho_{1}\right)=\inf _{H} D_{H}\left(\rho_{0}, \rho_{1}\right)
$$

where the infimum is taken over all Hamiltonians $H$ for which the boundary value problem in Equation (1) has a solution. The dynamic distance measure is defined for each pair of isospectral density 
operators because any two such can be connected by a solution to some von Neumann equation. The main result of this paper is that the dynamic distance measure is a proper distance measure on $\mathcal{D}(\sigma)$ :

Theorem 1. The dynamic distance measure is a proper distance measure.

Recall that a proper distance measure is a binary function dist that satisfies the following conditions:

Positivity: $\operatorname{dist}\left(\rho_{0}, \rho_{1}\right) \geq 0$

Non-degeneracy: $\operatorname{dist}\left(\rho_{0}, \rho_{1}\right)=0 \Longleftrightarrow \rho_{0}=\rho_{1}$

Symmetry: $\operatorname{dist}\left(\rho_{0}, \rho_{1}\right)=\operatorname{dist}\left(\rho_{1}, \rho_{0}\right)$

Triangle inequality: $\operatorname{dist}\left(\rho_{0}, \rho_{2}\right) \leq \operatorname{dist}\left(\rho_{0}, \rho_{1}\right)+\operatorname{dist}\left(\rho_{1}, \rho_{2}\right)$

One can show that the dynamic distance measure is also unitarily invariant:

$$
D\left(U \rho_{0} U^{\dagger}, U \rho_{1} U^{\dagger}\right)=D\left(\rho_{0}, \rho_{1}\right)
$$

The proof of Theorem 1 will be based on a fairly involved mathematical setup.

\section{Standard Purification of Isospectral Mixed States}

A state is called pure if its density operator has rank 1. In quantum mechanics, especially quantum information theory, purification refers to the fact that every density operator can be thought of as representing the reduced state of a pure state. More precisely, if $\rho$ is a density operator acting on $\mathcal{H}$, and $\mathcal{K}$ is a Hilbert space of large enough dimension, then there is a normalized ket $|\Psi\rangle$ in $\mathcal{H} \otimes \mathcal{K}$ such that $\rho$ is the partial trace of $|\Psi\rangle\langle\Psi|$ with respect to $\mathcal{K}$. By the standard purification of density operators on $\mathcal{H}$ of rank at most $k$ we will mean the surjective map $\pi: \mathcal{S}\left(\mathcal{H} \otimes \mathbb{C}^{k *}\right) \rightarrow \mathcal{D}_{k}(\mathcal{H})$ defined by $\pi|\Psi\rangle=\operatorname{Tr}_{\mathbb{C}^{k *}}|\Psi\rangle\langle\Psi|$. Here, $\mathbb{C}^{k *}$ is the space of linear functionals on $\mathbb{C}^{k}$ and $\mathcal{S}\left(\mathcal{H} \otimes \mathbb{C}^{k *}\right)$ is the unit sphere in $\mathcal{H} \otimes \mathbb{C}^{k *}$. If we canonically identify $\mathcal{H} \otimes \mathbb{C}^{k *}$ with the space $\mathcal{L}\left(\mathbb{C}^{k}, \mathcal{H}\right)$ of linear maps from $\mathbb{C}^{k}$ to $\mathcal{H}$, equipped with the Hilbert-Schmidt inner product, then

$$
\pi(\Psi)=\Psi \Psi^{\dagger}
$$

Write $P(\sigma)$ for the diagonal $k \times k$ matrix that has $\sigma$ as its diagonal, and let $\mathcal{S}(\sigma)$ be the space of those $\Psi$ in $\mathcal{L}\left(\mathbb{C}^{k}, \mathcal{H}\right)$ that satisfies $\Psi^{\dagger} \Psi=P(\sigma)$ when $\Psi^{\dagger} \Psi$ is expressed as a matrix relative to the standard basis in $\mathbb{C}^{k}$. Then $\mathcal{S}(\sigma)$ is a subspace of the unit sphere in $\mathcal{L}\left(\mathbb{C}^{k}, \mathcal{H}\right)$, and the standard purification in Equation (5) restricted to $S(\sigma)$ is a principal fiber bundle over $\mathcal{D}(\sigma)$ with right acting gauge group $\mathcal{U}(\sigma)$,

$$
U \cdot \Psi=\Psi U, \quad U \in \mathcal{U}(\sigma), \quad \Psi \in \mathcal{S}(\sigma)
$$

consisting of those unitaries in $\mathcal{U}(k)$ that commutes with $P(\sigma)$. The following two special cases are well known.

Example 1. If $\sigma=(1)$, then $\mathcal{D}(\sigma)$ is the complex projective $n$-space, $\mathcal{S}(\sigma)$ is the $(2 n+1)$-dimensional unit sphere, and $\pi$ is the generalized Hopf bundle.

Example 2. If $\sigma=\left(\frac{1}{k}, \frac{1}{k}, \ldots, \frac{1}{k}\right)$, then $\mathcal{D}(\sigma)$ is the Grassmann manifold of k-planes in $\mathcal{H}, \mathcal{S}(\sigma)$ is the Stiefel manifold of $k$-frames in $\mathcal{H}$, and $\pi$ is the Stiefel bundle. 
The vertical and horizontal bundles over $\mathcal{S}(\sigma)$ are the subbundles $\operatorname{VS}(\sigma)=\operatorname{Ker} d \pi$ and $\operatorname{HS}(\sigma)=$ $\mathrm{VS}(\sigma)^{\perp}$ of the tangent bundle of $\mathcal{S}(\sigma)$. Here ${ }^{\perp}$ denotes orthogonal complement with respect to the Hilbert-Schmidt product. Vectors in $\operatorname{VS}(\sigma)$ and $\mathrm{H} \mathcal{S}(\sigma)$ are called vertical and horizontal, respectively, and a curve in $\mathcal{S}(\sigma)$ is called horizontal if its velocity vectors are horizontal. Recall that for every curve $\rho$ in $\mathcal{D}(\sigma)$ and every $\Psi_{0}$ in the fiber over the initial operator $\rho\left(t_{0}\right)$, there is a unique horizontal lift of $\rho$ to $\mathcal{S}(\sigma)$ that extends from $\Psi_{0}$ [7] (page 69, Prop 3.1). For convenience, we assume that all curves in this paper are defined on a common unspecified interval $t_{0} \leq t \leq t_{1}$. Moreover, we assume that they are piecewise smooth.

The infinitesimal generators of the gauge group action in Equation (6) yield canonical isomorphisms between the Lie algebra $\mathfrak{u}(\sigma)$ of $\mathcal{U}(\sigma)$ and the fibers in $\operatorname{VS}(\sigma)$. The Lie algebra consists of all antiHermitian $k \times k$ matrices that commutes with $P(\sigma)$, and the isomorphisms are

$$
\mathfrak{u}(\sigma) \ni \xi \mapsto \Psi \xi \in \mathrm{V}_{\Psi} \mathcal{S}(\sigma)
$$

Furthermore, $\mathrm{H} \mathcal{S}(\sigma)$ is the kernel bundle of the gauge invariant mechanical connection form $\mathcal{A}_{\Psi}=$ $\mathbb{I}_{\Psi}^{-1} J_{\Psi}$, where $\mathbb{I}_{\Psi}: \mathfrak{u}(\sigma) \rightarrow \mathfrak{u}(\sigma)^{*}$ and $J_{\Psi}: \mathrm{T}_{\Psi} \mathcal{S}(\sigma) \rightarrow \mathfrak{u}(\sigma)^{*}$ are the locked inertia tensor and moment map, respectively,

$$
\mathbb{I}_{\Psi} \xi \cdot \eta=\frac{1}{2} \operatorname{Tr}\left(\left(\xi^{\dagger} \eta+\eta^{\dagger} \xi\right) P(\sigma)\right), \quad J_{\Psi}(X) \cdot \xi=\frac{1}{2} \operatorname{Tr}\left(X^{\dagger} \Psi \xi+\xi^{\dagger} \Psi^{\dagger} X\right)
$$

Using Equation (8) we can derive an explicit formula for the connection form. Indeed, if $m_{1}, m_{2}, \ldots, m_{l}$ are the multiplicities of the different eigenvalues in $\sigma$, with $m_{1}$ being the multiplicity of the greatest eigenvalue, $m_{2}$ the multiplicity of the second greatest eigenvalue, etc., and if for $j=1,2, \ldots, l$,

$$
E_{j}=\operatorname{diag}\left(\mathbf{0}_{m_{1}}, \ldots, \mathbf{0}_{m_{j-1}}, \mathbf{1}_{m_{j}}, \mathbf{0}_{m_{j+1}}, \ldots, \mathbf{0}_{m_{l}}\right)
$$

then

$$
\begin{aligned}
\mathbb{I}_{\Psi}\left(\sum_{j} E_{j} \Psi^{\dagger} X E_{j} P(\sigma)^{-1}\right) \cdot \xi & =\frac{1}{2} \operatorname{Tr}\left(\left(\sum_{j} P(\sigma)^{-1} E_{j} X^{\dagger} \Psi E_{j} \xi+\xi^{\dagger} \sum_{j} E_{j} \Psi^{\dagger} X E_{j} P(\sigma)^{-1}\right) P(\sigma)\right) \\
& =\frac{1}{2} \operatorname{Tr}\left(\sum_{j} E_{j}\left(X^{\dagger} \Psi \xi+\xi^{\dagger} \Psi^{\dagger} X\right) E_{j}\right) \\
& =\frac{1}{2} \operatorname{Tr}\left(X^{\dagger} \Psi \xi+\xi^{\dagger} \Psi^{\dagger} X\right) \\
& =J_{\Psi}(X) \cdot \xi
\end{aligned}
$$

for every $X$ in $\mathrm{T}_{\Psi} \mathcal{S}(\sigma)$ and every $\xi$ in $\mathfrak{u}(\sigma)$. Hence

$$
\mathcal{A}_{\Psi}(X)=\sum_{j} E_{j} \Psi^{\dagger} X E_{j} P(\sigma)^{-1}
$$

Observe that the orthogonal projection of $\mathrm{T}_{\Psi} \mathcal{S}(\sigma)$ onto $\mathrm{V}_{\Psi} \mathcal{S}(\sigma)$ is given by the connection form followed by the identification in Equation (7). Therefore, the vertical and horizontal projections of 
$X$ in $\mathrm{T}_{\Psi} \mathcal{S}(\sigma)$ are $X^{\perp}=\Psi \mathcal{A}_{\Psi}(X)$ and $X^{\|}=X-\Psi \mathcal{A}_{\Psi}(X)$, respectively. We finish this section with a discussion on the distance between the fibers of $\pi$ over two given density operators $\rho_{0}$ and $\rho_{1}$ in $\mathcal{D}(\sigma)$.

Consider the space $\Omega\left(\rho_{0}, \rho_{1}\right)$ of piecewise smooth curves that start in the fiber $\pi^{-1}\left(\rho_{0}\right)$ and end in the fiber $\pi^{-1}\left(\rho_{1}\right)$. This space can be given a natural smooth structure such that the tangent space at a curve $\Psi$ consists of all smooth vector fields $\chi$ along $\Psi$ that are vertical at the end points of $\Psi$. Let $E$ be the energy functional on $\Omega\left(\rho_{0}, \rho_{1}\right)$,

$$
E[\Psi]=\frac{1}{2} \int_{t_{0}}^{t_{1}} \operatorname{Tr}\left(\dot{\Psi}^{\dagger} \dot{\Psi}\right) \mathrm{d} t
$$

The differential of $E$ at $\Psi$ is given by

$$
d E[\Psi] \chi=\frac{1}{2}\left[\operatorname{Tr}\left(\chi^{\dagger} \dot{\Psi}+\dot{\Psi}^{\dagger} \chi\right)\right]_{t_{0}}^{t_{1}}-\frac{1}{2} \int_{t_{0}}^{t_{1}} \operatorname{Tr}\left(\chi^{\dagger} \nabla_{t} \dot{\Psi}+\nabla_{t} \dot{\Psi}^{\dagger} \chi\right) \mathrm{d} t
$$

where $\nabla_{t} \dot{\Psi}$ denotes the covariant derivative of $\dot{\Psi}$ along $\Psi$. We call $\Psi$ an extremal for $E$ if $d E[\Psi]=0$. Clearly, extremals for $E$ are geodesics: $\nabla_{t} \dot{\Psi}=0$.

The length of a curve $\Psi$ in $\mathcal{S}(\sigma)$ is

$$
L[\Psi]=\int_{t_{0}}^{t_{1}} \sqrt{\operatorname{Tr}\left(\dot{\Psi}^{\dagger} \dot{\Psi}\right)} \mathrm{d} t
$$

Moreover, the distance between $\pi^{-1}\left(\rho_{0}\right)$ and $\pi^{-1}\left(\rho_{1}\right)$ is defined as the infimum of the lengths of all curves in $\Omega\left(\rho_{0}, \rho_{1}\right)$. There is at least one curve in $\Omega\left(\rho_{0}, \rho_{1}\right)$ whose length equals the distance between the two fibers. This is because the fibers are compact. In addition, each such curve is an extremal for $E$. Therefore they are horizontal:

Proposition 2. If $\Psi$ is a geodesic in $\mathcal{S}(\sigma)$, then $J_{\Psi}(\dot{\Psi})$ is constant.

Proof. Choose any $\eta$ in $\mathfrak{u}(\sigma)$ and consider the variation $\Psi_{\varepsilon}(t)=\Psi(t) \exp (\varepsilon \eta)$. We have that $\operatorname{Tr}\left(\dot{\Psi}_{\varepsilon}^{\dagger} \dot{\Psi}_{\varepsilon}\right)=\operatorname{Tr}\left(\dot{\Psi}^{\dagger} \dot{\Psi}\right)$ since $\mathcal{U}(\sigma)$ acts through isometries. Hence

$$
\begin{aligned}
0 & =\frac{1}{2} \frac{\mathrm{d}}{\mathrm{d} \varepsilon}\left[\int_{\tau_{0}}^{\tau_{1}} \operatorname{Tr}\left(\dot{\Psi}_{\varepsilon}^{\dagger} \dot{\Psi}_{\varepsilon}\right) \mathrm{d} t\right]_{\varepsilon=0} \\
& =\frac{1}{2}\left[\operatorname{Tr}\left(\eta^{\dagger} \Psi^{\dagger} \dot{\Psi}+\dot{\Psi}^{\dagger} \Psi \eta\right)\right]_{\tau_{0}}^{\tau_{1}}-\frac{1}{2} \int_{\tau_{0}}^{\tau_{1}} \operatorname{Tr}\left(\eta^{\dagger} \Psi^{\dagger} \nabla_{t} \dot{\Psi}+\nabla_{t} \dot{\Psi}^{\dagger} \Psi \eta\right) \mathrm{d} t \\
& =\left[J_{\Psi}(\dot{\Psi}) \cdot \eta\right]_{\tau_{0}}^{\tau_{1}}
\end{aligned}
$$

for any $t_{0} \leq \tau_{0} \leq \tau_{1} \leq t_{1}$. We conclude that $J_{\Psi}(\dot{\Psi})$ is constant.

\section{Properties of the Dynamic Distance Measure}

In this section we prove that $D\left(\rho_{0}, \rho_{1}\right)$ equals the distance between the fibers $\pi^{-1}\left(\rho_{0}\right)$ and $\pi^{-1}\left(\rho_{1}\right)$. Theorem 1 follows easily from this observation.

Proposition 3. Suppose $\rho$ solves Equation (1). Let $\Phi$ be a horizontal lift of $\rho$. Then $D_{H}\left(\rho_{0}, \rho_{1}\right) \geq L[\Phi]$. Moreover, $D_{H}\left(\rho_{0}, \rho_{1}\right)=L[\Phi]$ if $i \dot{\Phi}=H \Phi$. 
Lemma 4. We have that $\operatorname{Tr}\left(\xi^{2} P(\sigma)\right) \leq \operatorname{Tr}(\xi P(\sigma))^{2}$ for every $\xi$ in $\mathfrak{u}(\sigma)$, and $\operatorname{Tr}\left(\xi^{2} P(\sigma)\right)=\operatorname{Tr}(\xi P(\sigma))^{2}$ if and only if $\xi$ is a constant multiple of the identity.

Proof. Write $i \xi=U \delta U^{\dagger}$, where $\delta$ is a real diagonal matrix and $U$ belongs to $\mathcal{U}(\sigma)$. We have that $\operatorname{Tr}\left(\xi^{2} P(\sigma)\right)=-\operatorname{Tr}\left(\delta^{2} P(\sigma)\right)$ and $\operatorname{Tr}(\xi P(\sigma))^{2}=-\operatorname{Tr}(\delta P(\sigma))^{2}$ since $P(\sigma)$ commutes with $U$. Moreover, $\operatorname{Tr}(\delta P(\sigma))^{2} \leq \operatorname{Tr}\left(\delta^{2} P(\sigma)\right)$, and $\operatorname{Tr}(\delta P(\sigma))^{2}=\operatorname{Tr}\left(\delta^{2} P(\sigma)\right)$ if and only if $\delta$ is a constant multiple of the identity. This is because $x \mapsto x^{2}$ is strongly convex.

Proof of Proposition 3. Let $\Phi$ be a horizontal lift of $\rho$, and let $\Psi$ be any lift of $\rho$ such that $i \dot{\Psi}=H \Psi$ and $\Psi\left(t_{0}\right)=\Phi\left(t_{0}\right)$. Then

$$
\Phi=\Psi U, \quad U(t)=\exp _{+}\left(-\int_{t_{0}}^{t} \mathcal{A}_{\Psi}(\dot{\Psi}) \mathrm{d} t\right)
$$

where $\exp _{+}$is the positive time-ordered exponential. Now,

$$
\begin{aligned}
\operatorname{Tr}\left(H^{2} \rho\right)-\operatorname{Tr}(H \rho)^{2} & =\operatorname{Tr}\left(\Psi^{\dagger} H^{2} \Psi\right)-\operatorname{Tr}\left(\Psi^{\dagger} H \Psi\right)^{2} \\
& =\operatorname{Tr}\left(\dot{\Psi}^{\dagger} \dot{\Psi}\right)+\operatorname{Tr}\left(\Psi^{\dagger} \dot{\Psi}\right)^{2} \\
& =\operatorname{Tr}\left(\dot{\Psi}^{\dagger} \dot{\Psi}\right)+\operatorname{Tr}\left(E_{j} \Psi^{\dagger} \dot{\Psi} E_{j}\right)^{2} \\
& =\operatorname{Tr}\left(\dot{\Psi}^{\dagger} \dot{\Psi}\right)+\operatorname{Tr}\left(\mathcal{A}_{\Psi}(\dot{\Psi}) P(\sigma)\right)^{2}
\end{aligned}
$$

and

$$
\begin{aligned}
\operatorname{Tr}\left(\dot{\Phi}^{\dagger} \dot{\Phi}\right) & =\operatorname{Tr}\left(U^{\dagger}\left(\dot{\Psi}^{\dagger}+\mathcal{A}_{\Psi}(\dot{\Psi}) \Psi^{\dagger}\right)\left(\dot{\Psi}-\Psi \mathcal{A}_{\Psi}(\dot{\Psi})\right) U\right) \\
& =\operatorname{Tr}\left(\dot{\Psi}^{\dagger} \dot{\Psi}+\left(\Psi^{\dagger} \dot{\Psi}-\dot{\Psi}^{\dagger} \Psi\right) \mathcal{A}_{\Psi}(\dot{\Psi})-\mathcal{A}_{\Psi}(\dot{\Psi})^{2} P(\sigma)\right) \\
& =\operatorname{Tr}\left(\dot{\Psi}^{\dagger} \dot{\Psi}\right)+2 \operatorname{Tr}\left(\Psi^{\dagger} \dot{\Psi} \mathcal{A}_{\Psi}(\dot{\Psi})\right)-\operatorname{Tr}\left(\mathcal{A}_{\Psi}(\dot{\Psi})^{2} P(\sigma)\right) \\
& =\operatorname{Tr}\left(\dot{\Psi}^{\dagger} \dot{\Psi}\right)+2 \operatorname{Tr}\left(\Psi^{\dagger} \dot{\Psi} E_{j} \Psi^{\dagger} \dot{\Psi} E_{j} P(\sigma)^{-1}\right)-\operatorname{Tr}\left(\mathcal{A}_{\Psi}(\dot{\Psi})^{2} P(\sigma)\right) \\
& =\operatorname{Tr}\left(\dot{\Psi}^{\dagger} \dot{\Psi}\right)+2 \operatorname{Tr}\left(\left(E_{j} \Psi^{\dagger} \dot{\Psi} E_{j}\right)^{2} P(\sigma)^{-1}\right)-\operatorname{Tr}\left(\mathcal{A}_{\Psi}(\dot{\Psi})^{2} P(\sigma)\right) \\
& =\operatorname{Tr}\left(\dot{\Psi}^{\dagger} \dot{\Psi}\right)+\operatorname{Tr}\left(\mathcal{A}_{\Psi}(\dot{\Psi})^{2} P(\sigma)\right)
\end{aligned}
$$

Hence

$$
\begin{aligned}
\operatorname{Tr}\left(H^{2} \rho\right)-\operatorname{Tr}(H \rho)^{2} & =\operatorname{Tr}\left(\dot{\Psi}^{\dagger} \dot{\Psi}\right)+\operatorname{Tr}\left(\mathcal{A}_{\Psi}(\dot{\Psi}) P(\sigma)\right)^{2} \\
& \geq \operatorname{Tr}\left(\dot{\Psi}^{\dagger} \dot{\Psi}\right)+\operatorname{Tr}\left(\mathcal{A}_{\Psi}(\dot{\Psi})^{2} P(\sigma)\right) \\
& =\operatorname{Tr}\left(\dot{\Phi}^{\dagger} \dot{\Phi}\right)
\end{aligned}
$$

by Lemma 4 . We conclude that $D_{H}\left(\rho_{0}, \rho_{1}\right) \geq L[\Phi]$. Moreover, if $\Psi$ is horizontal, and thus $\Psi=\Phi$, then $\operatorname{Tr}\left(H^{2} \rho\right)-\operatorname{Tr}(H \rho)^{2}=\operatorname{Tr}\left(\dot{\Phi}^{\dagger} \dot{\Phi}\right)$ according to Equation (16). In this case $D_{H}\left(\rho_{0}, \rho_{1}\right)=L[\Phi]$.

Corollary 5. The dynamic distance between two density operators $\rho_{0}$ and $\rho_{1}$ in $\mathcal{D}(\sigma)$ equals the distance between the fibers $\pi^{-1}\left(\rho_{0}\right)$ and $\pi^{-1}\left(\rho_{1}\right)$. 
Proof. Immediate from Proposition 3 and the fact that every curve in $\mathcal{S}(\sigma)$ is the solution to some Schrödinger equation. This is because the unitary group of $\mathcal{H}$ acts transitively on $\mathcal{S}(\sigma)$.

Proposition 3 and Corollary 5 shows that for any pair of density operators $\rho_{0}$ and $\rho_{1}$ in $\mathcal{D}(\sigma)$, there is a curve $\Psi$ in $\mathcal{S}(\sigma)$ that extends from the fiber over $\rho_{0}$, ends in the fiber over $\rho_{1}$, and for which $D\left(\rho_{0}, \rho_{1}\right)=$ $L[\Psi]$. This observation makes the proof of Theorem 1 fairly straightforward:

Proof of Theorem 1. The function $D$ is positive because $D_{H}\left(\rho_{0}, \rho_{1}\right)$ is always a non-negative number by Equation (2). Moreover, $D$ is non-degenerate. Indeed, let $\Psi$ be a curve in $\Omega\left(\rho_{0}, \rho_{1}\right)$ such that $D\left(\rho_{0}, \rho_{1}\right)=$ $L[\Psi]$. If $D\left(\rho_{0}, \rho_{1}\right)=0$, then $\Psi$ is stationary, and hence $\rho_{0}=\rho_{1}$. The opposite implication is obvious.

To see that $D$ is symmetric let $\Psi$ be a curve like the one in the proof of non-degeneracy. Define $\Phi$ by $\Phi(t)=\Psi\left(t_{1}+t_{0}-t\right)$. Then $\Phi$ is a horizontal curve that projects onto a curve in $\mathcal{D}(\sigma)$ from $\rho_{1}$ to $\rho_{0}$. Consequently, $D\left(\rho_{1}, \rho_{0}\right) \leq L[\Phi]=L[\Psi]=D\left(\rho_{0}, \rho_{1}\right)$. An identical argument shows that $D\left(\rho_{0}, \rho_{1}\right) \leq D\left(\rho_{1}, \rho_{0}\right)$. Thus, $D$ is symmetric.

Finally, to see that $D$ satisfies the triangle inequality let $\Psi_{i j}$ be a horizontal curve in $\mathcal{S}(\sigma)$ covering a curve in $\mathcal{D}(\sigma)$ from $\rho_{i}$ to $\rho_{j}, i, j=0,1,2$. Also assume that $D\left(\rho_{i}, \rho_{j}\right)=L\left[\Psi_{i j}\right]$. Then

$$
\Phi(t)=\left\{\begin{array}{lll}
\Psi_{01}\left(2 t-t_{0}\right), & \text { if } \quad t_{0} \leq t \leq\left(t_{1}+t_{0}\right) / 2 \\
\Psi_{12}\left(2 t-t_{1}\right), & \text { if } \quad\left(t_{1}+t_{0}\right) / 2 \leq t \leq t_{1}
\end{array}\right.
$$

is a horizontal curve connecting the fibers over $\rho_{0}$ and $\rho_{2}$. Therefore,

$$
D\left(\rho_{0}, \rho_{2}\right) \leq L[\Phi]=L\left[\Psi_{01}\right]+L\left[\Psi_{12}\right]=D\left(\rho_{0}, \rho_{1}\right)+D\left(\rho_{1}, \rho_{2}\right)
$$

Thus, $D$ satisfies the triangle inequality.

\section{Dynamic Distance Between Distinguishable Mixed States}

Assume the dimension of $\mathcal{H}$ is not less than twice the length of $\sigma$. Let $\rho_{0}$ and $\rho_{1}$ be density operators in $\mathcal{D}(\sigma)$ that represent distinguishable states in the sense that the fidelity between $\rho_{0}$ and $\rho_{1}$ vanishes. Furthermore, let $\Psi_{0}$ and $\Psi_{1}$ be any purifications of $\rho_{0}$ and $\rho_{1}$, and define a curve $\Psi=\Psi(t)$ by

$$
\Psi(t)=\cos t \Psi_{0}+\sin t \Psi_{1}, \quad 0 \leq t \leq \pi / 2
$$

The curve $\Psi$ is a distance minimizing geodesic when regarded a curve in the unit sphere in $\mathcal{L}\left(\mathbb{C}^{k}, \mathcal{H}\right)$, and as such it has length $\pi / 2$. Accordingly, $D\left(\rho_{0}, \rho_{1}\right) \geq \pi / 2$. On the other hand, $\Psi$ is contained in $\mathcal{S}(\sigma)$ because the supports of $\Psi_{0}$ and $\Psi_{1}$ are orthogonal:

$$
\Psi_{0}^{\dagger} \Psi_{1}=\Psi_{1}^{\dagger} \Psi_{0}=0 \Longrightarrow \Psi(t)^{\dagger} \Psi(t)=\cos ^{2} t \Psi_{0}^{\dagger} \Psi_{0}+\sin t \cos t\left(\Psi_{0}^{\dagger} \Psi_{1}+\Psi_{0}^{\dagger} \Psi_{1}\right)+\sin ^{2} t \Psi_{1}^{\dagger} \Psi_{1}=P(\sigma)
$$

Hence, $D\left(\rho_{0}, \rho_{1}\right) \leq L[\Psi]=\pi / 2$. We conclude that

$$
D\left(\rho_{0}, \rho_{1}\right)=\pi / 2
$$

A Hamiltonian $H$ that transports $\rho_{0}$ to $\rho_{1}$ in such a way that the $H$-distance between $\rho_{0}$ and $\rho_{1}$ is equal to the dynamic distance can be obtained as follows. The sequence

$$
\frac{1}{\sqrt{p_{1}}} \Psi_{0} e_{1}, \frac{1}{\sqrt{p_{1}}} \Psi_{1} e_{1}, \frac{1}{\sqrt{p_{2}}} \Psi_{0} e_{2}, \frac{1}{\sqrt{p_{2}}} \Psi_{1} e_{2}, \ldots, \frac{1}{\sqrt{p_{k}}} \Psi_{0} e_{k}, \frac{1}{\sqrt{p_{k}}} \Psi_{1} e_{k}
$$


where $e_{j}$ is the $j^{\text {th }}$ standard basis vector in $\mathbb{C}^{k}$, is an orthonormal $2 k$-frame in $\mathcal{H}$. Let $H$ be any Hamiltonian that satisfies

$$
H \Psi_{0} e_{j}=i \Psi_{1} e_{j}, \quad H \Psi_{1} e_{j}=-i \Psi_{0} e_{j}, \quad j=1,2, \ldots k
$$

Then $\Psi$ is the solution to the Schrödinger equation of $H$ with initial value $\Psi_{0}$.

\section{Relation Between the Dynamic Distance Measure and Bures Distance}

Suppose $\mathcal{H}$ is $n$-dimensional. Let $\mathcal{S}_{\text {inv }}\left(\mathbb{C}^{n}, \mathcal{H}\right)$ be the space of all invertible maps in $\mathcal{L}\left(\mathbb{C}^{n}, \mathcal{H}\right)$ with unit norm, and $\mathcal{D}_{\text {inv }}(\mathcal{H})$ be the space of all invertible density operators acting on $\mathcal{H}$. Then $\Pi: \mathcal{S}_{\text {inv }}\left(\mathbb{C}^{n}, \mathcal{H}\right) \rightarrow \mathcal{D}_{\text {inv }}(\mathcal{H})$ defined by $\Pi(\Psi)=\Psi \Psi^{\dagger}$ is a $\mathcal{U}(n)$-bundle, which we call Uhlmann's bundle since it first appeared in [6]. The geometry of Uhlmann's bundle has been thoroughly investigated, and it is an important tool in quantum information theory, mainly due to its close relationship with the Bures distance measure $[8,9]$.

Uhlmann's bundle is equipped with the mechanical connection, which means that the horizontal bundle is the orthogonal complement of the vertical bundle with respect to the Hilbert-Schmidt inner product. Moreover, the Bures distance between two density operators in $\mathcal{D}_{\text {inv }}(\mathcal{H})$ equals the distance between the corresponding fibers of $\Pi$, see [9]. We denote the Bures distance measure by $D_{B}$.

Suppose $\sigma$ has length $n$. Then $\mathcal{S}(\sigma)$ is a submanifold of $\mathcal{S}_{\text {inv }}\left(\mathbb{C}^{n}, \mathcal{H}\right)$. Moreover, the vertical bundle of $\mathcal{S}(\sigma)$ is subbundle of the restriction of the vertical bundle of $\mathcal{S}_{\text {inv }}\left(\mathbb{C}^{n}, \mathcal{H}\right)$ to $\mathcal{S}(\sigma)$. However, no nonzero horizontal vector in Uhlmann's bundle is tangential to $\mathcal{S}(\sigma)$. To see this, let $\Psi$ be any element in $\mathcal{S}(\sigma)$. Then $X$ in $\mathrm{T}_{\Psi} \mathcal{S}_{\text {inv }}\left(\mathbb{C}^{n}, \mathcal{H}\right)$ is horizontal, i.e., is annihilated by the mechanical connection of the Uhlmann bundle, if and only if

$$
\Psi^{\dagger} X-X^{\dagger} \Psi=0
$$

see [6]. On the other hand, every $X$ in $\mathrm{T}_{\Psi} \mathcal{S}(\sigma)$ satisfies

$$
\Psi^{\dagger} X+X^{\dagger} \Psi=0
$$

since $\Psi^{\dagger} \Psi=P(\sigma)$. Clearly, only the zero vector satisfies both Equations (26) and (27).

The distance between $\rho_{0}$ and $\rho_{1}$ in $\mathcal{D}(\sigma)$ is never smaller than the Bures distance between them. Indeed, every curve between $\pi^{-1}\left(\rho_{0}\right)$ and $\pi^{-1}\left(\rho_{1}\right)$ in $\mathcal{S}(\sigma)$ is a curve between $\Pi^{-1}\left(\rho_{0}\right)$ and $\Pi^{-1}\left(\rho_{1}\right)$ in $\mathcal{S}_{\text {inv }}\left(\mathbb{C}^{n}, \mathcal{H}\right)$, and since the metrics on the total spaces of the two bundles are induced from a common ambient metric we can conclude that

$$
D\left(\rho_{0}, \rho_{1}\right) \geq D_{B}\left(\rho_{0}, \rho_{1}\right)
$$

Uhlmann [9] and Dittmann [10,11] have derived explicit formulas for the Bures distance for density operators on finite dimensional Hilbert spaces. For density operators on $\mathbb{C}^{2}$ the formula reads

$$
D_{B}(\rho, \rho+\delta \rho)^{2}=\frac{1}{4} \operatorname{Tr}\left(\delta \rho \delta \rho+\frac{1}{\operatorname{det} \rho}(\delta \rho-\rho \delta \rho)^{2}\right)
$$


We use this formula to show that there are density operators $\rho_{0}$ and $\rho_{1}$ acting on $\mathbb{C}^{2}$ for which the inequality in Equation (28) is strict.

Suppose $\sigma=\left(p_{1}, p_{2}\right)$, let $\varepsilon>0$, and define a curve $\Psi$ in $\mathcal{S}(\sigma)$ by

$$
\Psi(t)=\left[\begin{array}{cc}
\sqrt{p_{1}} \cos (\varepsilon t) & \sqrt{p_{2}} \sin (\varepsilon t) \\
-\sqrt{p_{1}} \sin (\varepsilon t) & \sqrt{p_{2}} \cos (\varepsilon t)
\end{array}\right], \quad 0 \leq t \leq 1
$$

Set $\rho_{0}=\Psi(0) \Psi(0)^{\dagger}$ and $\rho_{1}=\Psi(1) \Psi(1)^{\dagger}$. Then, for $\varepsilon$ small enough, the length of $\Psi$ equals $D\left(\rho_{0}, \rho_{1}\right)$. In this case, $D\left(\rho_{0}, \rho_{1}\right)=L[\Psi]=\varepsilon$. However, Equation (29) yields

$$
D_{B}\left(\rho_{0}, \rho_{1}\right)=\frac{p_{1}-p_{2}}{\sqrt{2}}|\sin \varepsilon| \sqrt{2+\frac{\left(p_{1}-p_{2}\right)^{2}}{2 p_{1} p_{2}} \sin ^{2} \varepsilon}
$$

\section{Conclusion}

In summary, we have introduced a measurable quantity called the dynamic distance measure on each space of isospectral density operators, and shown that it is a proper distance measure, i.e., a positive, non-degenerate, symmetric binary function that satisfies the triangle inequality. The main result was formulated in Section 2, but its proof was postponed until Section 4 to make the paper accessible also to those readers who are mainly interested in the result rather than the extensive geometrical setup and fairly technical proof. We have also compared our dynamic distance measure with the Bures distance. The outcome of that comparison is that the dynamic distance measure and the Bures distance are different. In fact, the dynamic distance measure is bounded from below by the Bures distance. Because the dynamic distance measure is defined in terms of Hamiltonians we believe that our results have many interesting applications in fields such as quantum computing and condense matter, where Hamiltonians for specific quantum operations or specific quantum systems are usually defined explicitly.

\section{Acknowledgements}

The second author acknowledges the financial support from the Swedish Research Council (VR).

\section{Conflicts of Interest}

The authors declare no conflict of interest.

\section{References}

1. Ohya, M.; Volovich, I. Mathematical Foundations of Quantum Information and Computation and Its Applications to Nano- and Bio-systems; Springer: New York, NY, USA, 2011.

2. Sharif, P.; Heydari, H. An introduction to multi-player, multi-choice quantum games. In Econophysics of Systemic Risk and Network Dynamics; Abergel, F. Chakrabarti, B.K., Chakraborti, A., Ghosh, A., Eds.; Springer: Milan, Italy, 2012; pp. 217-237. 
3. Landsburg, S.E. Quantum Game Theory. In Wiley Encyclopedia of Operations Research and Management Science; Cochran, J.J., Cox, L.A., Keskinocak, P., Kharoufeh, J.P., Smith, J.C., Eds.; Wiley: Hoboken, NJ, USA, 2011.

4. Nielsen, M.A.; Chuang, I.L. Quantum Computation and Quantum Information; Cambridge University Press: Cambridge, UK, 2010.

5. Uhlmann, A. Parallel transport and "quantum holonomy" along density operator. Rep. Math. Phys. 1986, 74, 229-240.

6. Uhlmann, A. A gauge field governing parallel transport along mixed states. Lett. Math. Phys. 1991, 21, 229-236.

7. Kobayashi, S.; Nomizu, K. Foundations of differential geometry. Volume I; Wiley Classics Library; Wiley: New York, NY, USA, 1996.

8. Bures, D. An extension of Kakutani's theorem on infinite product measures to the tensor product of semifinite $\mathrm{w}^{*}$-algebras. Trans. Am. Math. Soc. 1969, 135, 199-212.

9. Uhlmann, A. The metric of Bures and the geometric phase. In Groups and Related Topics; Gielerak, R., Lukierski, J., Popowicz, Z., Eds.; Mathmatics Physics Studies, Volume 13; Kluwer: Dordrecht, The Netherlands, 1992; pp. 267-274.

10. Dittmann, J. On the Riemannian geometry of finite dimensional mixed states. Sem. S. Lie 1993, 3, 73-87.

11. Dittmann, J. Explicit formulae for the Bures metric. J. Phys. A: Math. Gen. 1999, $32,2663-2670$.

(c) 2013 by the authors; licensee MDPI, Basel, Switzerland. This article is an open access article distributed under the terms and conditions of the Creative Commons Attribution license (http://creativecommons.org/licenses/by/3.0/). 